\title{
Failure to Elicit Near-Death Experiences in Induced Cardiac Arrest
}

\author{
Bruce Greyson, M.D. \\ University of Virginia, Charlottesville, VA \\ Janice M. Holden, Ed.D. \\ University of North Texas, Denton, TX \\ J. Paul Mounsey, Ph.D., M.B.B.Ch. \\ University of Virginia, Charlottesville, VA
}

ABSTRACT: Persons reporting near-death experiences (NDEs) sometimes describe a sense of having been out of their bodies and observing their surroundings from a visual perspective outside of and above their bodies. We attempted to study such phenomena during the surgical implantation of automatic implantable cardioverters/defibrillators (ICDs), electrical devices that monitor the patient's heartbeat and automatically detect cardiac arrest and administer an electrical shock to return the heart to normal rhythm. When ICDs are implanted in a patient's chest, cardiac arrest is induced under closely monitored conditions, in order to test the ICD's sensitivity and effectiveness. This study was designed to investigate the accuracy of out-of-body perceptions during NDEs that occur during these induced cardiac arrests. A computer in the operating room displayed quasi-randomly-selected unusual visual targets so that they were visible only from above eye level, from a visual perspective looking down upon the body of the unconscious patient. In a series of 52 induced cardiac arrests, no patient reported having had a near-death experience, and none reported a sense of having left the physical body or observing from an outof-body visual perspective. This failure to find a single NDE in 52 induced cardiac arrests may have been due to preoperative reassurances that patients

Bruce Greyson, M.D., is the Chester F. Carlson Professor of Psychiatry and Neurobehavioral Sciences and Director of the Division of Perceptual Studies at the University of Virginia. Janice M. Holden, Ed.D., is Professor of Counseling and Interim Chair of the Department of Counseling, Higher Education, and Early Childhood at the University of North Texas College of Education. J. Paul Mounsey, Ph.D., M.B.B.Ch., is Associate Professor of Internal Medicine in the Division of Cardiology at the University of Virginia. This research was supported by Grant Number 35/02 from the Bial Foundation. Reprint requests should be addressed to Dr. Greyson at the Division of Perceptual Studies, University of Virginia Health System, P.O. Box 800152, Charlottesville, VA 22908-0152; e-mail: cbg4d@virginia.edu. 
would not be in danger of dying, the brief duration of the induced cardiac arrest, or the amnestic effects of pre-anesthetic sedative medication.

Near-death experiences (NDEs) are profound subjective experiences reported by between 9 percent and 18 percent of patients who have been in a life-threatening situation (Greyson, 1998). NDEs are commonly reported by survivors of cardiac arrest (Greyson, 2003; Parnia, Waller, Yeates, and Fenwick, 2001; Schoenbeck and Hocutt, 1991; Schwaninger, Eisenberg, Schechtman, and Weiss, 2002; van Lommel, van Wees, Meyers, and Elfferich, 2001). Previous studies of NDEs in patients with cardiac arrest have included anecdotal accounts of patients claiming to have left their physical bodies during resuscitation procedures and describing those procedures as if they had observed them from a visual perspective near the ceiling of the room (Holden, 1988; Ring and Lawrence, 1993). For example, Emily Cook, Bruce Greyson, and Ian Stevenson (1998) reported a case in which a 56-year-old man, upon regaining consciousness after quadruple bypass surgery, told his cardiologist that, from a position above the operating room during the surgery, he had seen one of the surgeons "flapping his arms as if trying to fly" (p. 399). The surgeon later explained that it was his practice, from the time he "scrubbed in" to the time he actually touched the patient, to avoid contaminating his hands by holding them against his chest and pointing with his elbows to give instructions to operating room personnel. Cardiologists have been reporting such anecdotes for at least a quarter century (e.g., Sabom, 1982). However, none of the previous studies has included a controlled test of the accuracy of those observations, because of the unpredictable occurrence of NDEs in those settings (Parnia, Waller, Yeates, and Fenwick, 2001; Schoenbeck and Hocutt, 1991; Schwaninger, Eisenberg, Schechtman, and Weiss, 2002; van Lommel, van Wees, Meyers, and Elfferich, 2001).

Regarding the objects perceived during NDEs, our preliminary research (Holden, 1988, 1989) and published NDE accounts have indicated that visual perception while the $\mathrm{NDEr}$ is allegedly perceiving the material world is equivalent to physical vision in several respects: perception of color, shape, and movement; object recognition; ability to read both printed text and electronic displays; and attention-getting factors of brightness and movement. In addition, NDErs have reported remembering both seemingly meaningful phenomena, such as seeing medical personnel rushing in to re- 
suscitate or seeing readings of their vital signs on electronic monitors, as well as seemingly meaningless phenomena, such as dust on top of a light fixture or a nurse's plaid shoelaces (Ring and Lawrence, 1993).

\section{Implantable Cardioverters/Defibrillators}

The cardiac electrophysiology clinic provides a unique opportunity in which to assess the accuracy of patients' claims to observe their own resuscitation, because the cardiac arrests are induced in a planned and controlled environment. Patients who have experienced sustained ventricular arrhythmias and who are at high risk for sudden cardiac death are often referred to cardiac electrophysiology clinics for surgical implantation and testing of implantable cardioverters/ defibrillators (ICDs). Patients who undergo implantation and testing of ICDs commonly have cardiac arrest induced under controlled circumstances, exposing them to the opportunity to experience NDEs under closely monitored conditions. Milne (1995) studied the incidence and correlates of NDEs in cardiac electrophysiology clinics at two hospitals. She found that 14 percent of the patients who experienced hemodynamic instability during the cardiac electrophysiology procedures reported NDEs, whereas none of the patients who did not experience hemodynamic instability reported NDEs.

In the University of Virginia Health System Electrophysiology Clinic, patients are often hospitalized overnight prior to ICD implantation. They are typically premedicated with midazolam and/or fentanyl, and receive anesthesia with propofol and/or etomidate during the procedure. The procedure takes approximately one hour, during which the patient is typically unconscious for about 10 minutes, during which cardiac arrest is induced in order to test and calibrate the ICD. Patients typically remain in the hospital overnight following the procedure and are discharged from the hospital the following day.

\section{Objectives}

We attempted to study patients' claims of accurate "out-of-body" perceptions during their near-death experiences through the use of unique visual targets strategically located in the electrophysiology clinic procedure room during implantation and testing of ICDs. Reports of NDEs during cardiac arrest have often been ignored or 
discounted because of skepticism as to whether the patients really suffered cardiac arrests or were otherwise near death. This study was designed to remove such doubts because the cardiac arrest was immediately observed and recorded.

Reports of accurate perceptions by unconscious patients while allegedly out of their bodies have often been ignored or discounted because the reported perceptions were of expected or easily guessed objects, and/or there was no truly independent corroboration of the accuracy of those perceptions. This study was designed to remove such doubts because the specific targets (1) were unexpected and unusual visual images, (2) were generated by a quasi-random computer algorithm, and (3) were compared to the pool of possible targets by three independent judges blind to the actual target.

We hypothesized that participants describing near-death experiences during ICD testing would be able to identify a randomly generated visual display that was visible from a height over their body at the time of the procedure. We attempted to test this hypothesis by asking all participants, regardless of whether or not they described NDEs occurring during their ICD testing, whether they remembered anything unusual happening during their procedure, and whether they saw anything unexpected during their procedure.

\section{Methods}

\section{Procedure for ICD Implantation and Testing}

The ICD implantation and testing was carried out by cardiac electrophysiologists and their clinical support personnel at the University of Virginia Health System in one of two procedure rooms designed for that purpose. Patients were premedicated with midazolam and/or fentanyl, and received anesthesia with propofol and/or etomidate during the procedure. The procedure took approximately one hour, during which the patient was typically unconscious for about 10 minutes. During that 10-minute period of unconsciousness, ventricular fibrillation was typically induced twice in order to test and calibrate the ICD. Physiological variables were routinely documented in the patients' medical record, including the duration of the cardiac arrest (both ventricular fibrillation and cardiac asystole) and medications administered during the procedure. 


\section{Visual Target}

Prior to the patients' entry into the procedure room for the ICD implantation, an Apple Macintosh Pismo Powerbook laptop computer was placed above eye level in the procedure room, attached to the top surface of a cabinet or video monitor. The computer was opened and laid flat, so that the computer screen, measuring $34 \mathrm{~cm} \times 24 \mathrm{~cm}$, faced the ceiling. The operating table on which the patient would lie was $0.9 \mathrm{~m}$ from the floor, and the height of the procedure room was $3.2 \mathrm{~m}$. The surface on which the computer was attached was approximately $1.4 \mathrm{~m}$ above the patient, and $0.9 \mathrm{~m}$ below the ceiling.

After the ICD was surgically implanted and immediately prior to the ICD testing, the computer was turned on. The computer program then selected using a quasi-random procedure and displayed 1 of 12 unexpected but easily-identifiable simple cartoon animations that was displayed in 1 of 5 randomly selected colors continuously during the ICD testing. In that way, the participant had 1 chance in 60 of guessing the correct target image and color.

The pool of 12 unexpected visual targets included easily recognized animated images, such as a butterfly floating, a frog jumping, and fireworks exploding above the Statue of Liberty. Each image was displayed in red, orange, green, yellow, or purple. The quasi-random algorithm for selecting the target animation and color was determined by the exact second at which the program was turned on. The target sequence consisted of the selected color spreading rapidly from the center of the computer monitor until it filled the screen. The screen then flashed three times with the name of the color spelled out in white letters against the colored background. The colored screen was then replaced by a bright yellow screen on which the current time was displayed. That screen was then replaced by a white screen against which the selected cartoon animation played in the color already designated. The pattern was then repeated rotated 180 degrees so that the animation could be identified from any spatial orientation. The entire sequence took 40 seconds, and was repeated continuously for an hour. The computer program kept a record of which target image and color were selected and displayed during each procedure. However, no one had access to that record or knew the identity of the selected and displayed visual targets until the conclusion of the study. 


\section{Debriefing Interview}

Patients typically remained in the hospital following the ICD implantation and testing and were discharged from the hospital the following day. After the electrophysiology clinic team determined that the patient had recovered sufficiently to be interviewed again, participants were given a 15 -minute debriefing interview by one of us (B.G.). The debriefing interview was tape recorded with participants' permission and included 5 questions about (1) what they recalled of the procedure, (2) whether they recalled losing consciousness, (3) what they recalled next after losing consciousness, (4) whether they recalled anything unusual happening during the procedure, and (5) whether they saw anything unusual in the room during the procedure. Those participants who did not spontaneously describe seeing the target computer screen above their body were asked if they saw one, and asked to describe any visual images they saw displayed. The debriefing interview also included the NDE Scale (Greyson, 1983), a standardized instrument for identifying and quantifying near-death experiences; and 2 questions asking (1) whether they had ever heard or read about such experiences and (2) what they thought about such experiences.

\section{Analysis of Data}

The hypothesis that participants who report NDEs would be able to describe accurately the quasi-randomly generated targets was to be tested by evaluating the participants' descriptions of the target (if any) from the debriefing interview. Descriptions of the target from each participant's tape-recorded debriefing interview would be compared to the entire pool of possible targets by all 3 co-investigators independently, who would rank each of the 12 possible targets and each of the 5 possible colors according to their similarity to the participant's description. After the judging had been completed and recorded, the computer's record of the correct targets would be accessed, and the correct targets compared with the rankings of the investigators. 


\section{Results}

A total of 25 patients participated in the study. Twenty-three of those had 2 induced cardiac arrests each, and 2 patients had 3 induced cardiac arrests each, for a total of 52 induced cardiac arrests.

\section{Patient Sociodemographic Characteristics}

The mean age of the patients was 66.0 years $(\mathrm{SD}=14.2)$, with a range from 39 to 87 years. The sample included 21 men ( 84 percent) and 4 women ( 16 percent); and 20 Euro-Americans ( 80 percent) and 5 African-Americans (20 percent). Their mean formal education was 12.3 years $(\mathrm{SD}=3.7$ ), with a range from 3 to 22 years.

Twenty-two patients (88 percent) described themselves as Protestants, 2 ( 8 percent) as Roman Catholics, and 1 (4 percent) as Wiccan. Ten patients ( 40 percent) described themselves as very religious, 6 ( 24 percent) as moderately religious, 7 (28 percent) as slightly religious, and 2 ( 8 percent) as not religious at all. Ten patients (40 percent) described themselves as very spiritual, 7 ( 28 percent) as moderately spiritual, 7 (28 percent) as slightly spiritual, and 1 (4 percent) as not spiritual at all.

\section{Patient Clinical Characteristics}

Underlying cardiac diagnoses for these patients included coronary artery disease in 18 patients (72 percent), nonischemic cardiomyopathy in 6 (24 percent), and other structural heart disease in 1 (4 percent). The indication for the ICD implantation was repeated sudden cardiac death in 14 patients (56 percent), syncopal ventricular tachycardia in 6 (24 percent), nonsyncopal ventricular tachycardia in 3 (12 percent), inducible sustained ventricular tachycardia in 1 (4 percent), and "other" in 1 ( 4 percent). Mean left ventricular ejection fraction in these patients was 26.8 percent $(S D=12.0)$, with a range from 7 percent to 51 percent. Canadian Cardiovascular Society Angina Class (Campeau, 1976) was severe (Class 4 ) for 8 patients ( 32 percent), marked (Class 3) for 10 (40 percent), moderate (Class 2) for 2 ( 8 percent), minimal (Class 1 ) for 2 ( 8 percent), and none for 3 (12 percent). The mean Cardiac Health Profile (Währborg and Emanuelsson, 1996) quality of life for this sample was 62.4 percent $(\mathrm{SD}=13.0)$ and $\mathrm{CHP}$ satisfaction was 89.4 percent $(\mathrm{SD}=19.2)$. 


\section{Procedure Details}

Due to delays in scheduling the implantation procedure secondary to patient complications and/or competing emergent procedures, the number of hours between the initial interview and the actual ICD implantation procedure ranged widely, from 3 to 257 . The mean time elapsed between the initial interview and the ICD implantation procedure was 30.0 hours (SD $=55.8$ ).

The actual procedure performed was ICD implantation for 13 patients ( 52 percent), biventricular pacemaker-defibrillator implantation for 10 (40 percent), ICD generator change for 1 (4 percent), and reposition of ICD leads for 1 (4 percent).

Mean doses of medication included midazolam $2.69 \mathrm{mg}(\mathrm{SD}=1.71)$, with a range from 0 to $6 \mathrm{mg}$; fentanyl $169.44 \mathrm{mg}(\mathrm{SD}=137.35)$, with a range from 0 to $600 \mathrm{mg}$; propafol $1.53 \mathrm{mg}(\mathrm{SD}=0.51)$, with a range from 0 to $2 \mathrm{mg}$; and etomidate $1.53 \mathrm{mg}(\mathrm{SD}=0.51)$, with a range from 0 to $2 \mathrm{mg}$.

The mean duration of ventricular fibrillation on the first test was 8.76 seconds $(S D=4.01$ ), with a range from 3.80 to 18.00 ; on the second test, 9.69 seconds ( $\mathrm{SD}=5.71$ ), with a range from 3.80 to 25.0 : and on the third induced ventricular fibrillation, 9.60 seconds for the two patients who had one, which lasted for 9.00 and 10.20 seconds. The mean duration of ventricular fibrillation for all 52 induced cardiac arrests was 9.22 seconds (4.72), with a range from 3.80 to 25.00 . The median duration was 8.00 seconds.

The mean time elapsed between the ICD implantation procedure and the debriefing interview was 13.4 hours $(S D=13.5)$, with a range from 1 to 61 hours.

\section{Patients' Recollections of the Procedure}

Thirteen patients (52 percent) acknowledged losing consciousness, 10 (40 percent) denied losing consciousness, and 2 ( 8 percent) were unsure whether they had lost consciousness. Twenty patients (80 percent) denied recall of any events during the period of unconsciousness, and 5 (20 percent) described some recall of events.

\section{Near-Death Experiences}

On the NDE Scale, none of the patients scored 7 points or higher, which would have indicated the occurrence of a near-death experience. 


\section{Table I}

Positive responses to NDE Scale items for duration of unconsciousness

\begin{tabular}{llll}
\hline \multicolumn{1}{c}{ Item } & Definite & Equivocal & None \\
\hline Cognitive features: & & & \\
Time distortion & $1(4 \%)$ & $0(0 \%)$ & $24(96 \%)$ \\
Thought acceleration & $0(0 \%)$ & $0(0 \%)$ & $25(100 \%)$ \\
Life review & $0(0 \%)$ & $0(0 \%)$ & $25(100 \%)$ \\
Sudden understanding & $0(0 \%)$ & $0(0 \%)$ & $25(100 \%)$ \\
Affective features: & & & \\
Peace & $1(4 \%)$ & $0(0 \%)$ & $24(96 \%)$ \\
Joy & $0(0 \%)$ & $0(0 \%)$ & $25(100 \%)$ \\
Cosmic unity & $0(0 \%)$ & $1(4 \%)$ & $24(96 \%)$ \\
Light & $0(0 \%)$ & $0(0 \%)$ & $25(100 \%)$ \\
Paranormal features: & & & \\
Vivid senses & $0(0 \%)$ & $0(0 \%)$ & $25(100 \%)$ \\
Extrasensory perception & $0(0 \%)$ & $0(0 \%)$ & $25(100 \%)$ \\
Precognitive visions & $0(0 \%)$ & $0(0 \%)$ & $25(100 \%)$ \\
Out-of-body experience & $0(0 \%)$ & $0(0 \%)$ & $25(100 \%)$ \\
Transcendental features: & & & \\
Unearthly realm & $0(0 \%)$ & $1(4 \%)$ & $24(96 \%)$ \\
Mystical presence & $0(0 \%)$ & $0(0 \%)$ & $25(100 \%)$ \\
Visible spirits & $0(0 \%)$ & $1(4 \%)$ & $24(96 \%)$ \\
Border & $0(0 \%)$ & $0(0 \%)$ & $25(100 \%)$ \\
\hline
\end{tabular}

For the approximately 10 -minute period of unconsciousness, 23 patients ( 92 percent) had a score of 0,1 patient ( 4 percent) had a score of 1 , and 1 patient (4 percent) had a score of 6 .

The mean NDE Scale score was $0.28(\mathrm{SD}=1.35)$ for the period of unconsciousness, with a range of 0 to 6 . Mean scores for the Cognitive Component were 0.08 (SD $=0.45$ ); for the Affective Component, 0.12 ( $\mathrm{SD}=0.67$ ); for the Paranormal Component, $0(\mathrm{SD}=0)$; and for the Transcendental Component, $0.08(\mathrm{SD}=0.31$ ).

The rates of positive, equivocal, and negative responses to specific items on the NDE Scale are presented in Table 1. One patient (4 percent) described cessation of time while unconscious, and 1 (4 percent) described a strong feeling of peacefulness. One additional patient ( 4 percent) described a sense of being no longer in conflict, 
which may be interpreted as an equivocal sense of cosmic unity; 1 (4 percent) reported a sense of being in an unfamiliar "place," which may be interpreted as an equivocal description of an "unearthly realm"; and 1 (4 percent) reported a sense of the possible presence of a deceased loved one, which may be interpreted as an equivocal description of a vision of a deceased spirit. No participants reported a sense of leaving the physical body, or other typical NDE features of thoughts speeding up, a life review, a sense of sudden understanding, a feeling of joy, an encounter with a light, sensory vividness, extrasensory perception, precognitive visions, encounter with a mystical presence or voice, or a border or "point of no return" while they were unconscious.

\section{Patients' Understanding of Near-Death Experiences}

Twenty patients ( 80 percent) said that prior to this study they had had some awareness of the phenomena of near-death experiences, and 5 (20 percent) said they had never heard of such things. When asked what they thought might explain near-death experiences, 13 patients (52 percent) described some spiritual explanation, 4 (16 percent) described a psychological explanation, 1 (4 percent) described a physiological explanation, and 7 ( 28 percent) stated they had no idea.

\section{Target Descriptions}

None of the patients described any visual imagery during the procedure. When told about the laptop computer and asked to guess its visual display, none would do so. When shown the 12 possible target symbols and asked to guess which one had been displayed, only 2 patients ( 8 percent) would do so, and both guessed incorrectly. When shown the 5 possible target colors and asked to guess which one had been displayed, only three patients ( 12 percent) would do so, of whom 1 guessed correctly.

\section{Discussion}

Evidence from this study that patients during cardiac arrest have perceptions that they could not have had normally from the position of their bodies would have had profound implications for the question of mind/brain relationships, and specifically would have provided evidence for the independent functioning of the mind while the brain 
was physiologically impaired. However, this study provided no such evidence, inasmuch as none of the patients reported a near-death experience, with or without perceptions from an apparent out-of-body perspective.

In light of the consistently reported finding that near-death experiences occur in 9 percent to 18 percent of spontaneous cardiac arrests (Greyson, 2003; Parnia, Waller, Yeates, and Fenwick, 2001; Schoenbeck and Hocutt, 1991; Schwaninger, Eisenberg, Schechtman, and Weiss, 2002; van Lommel, van Wees, Meyers, and Elfferich, 2001), we anticipated between 5 and 10 NDEs among our sample of 52 induced cardiac arrests. Milne's (1995) experience in cardiac electrophysiology clinics suggested that we might elicit 7 NDEs in this sample. Therefore, we were surprised to identify no such experiences among these 52 induced cardiac arrests. Our failure to elicit any neardeath experiences in this study precluded testing the accuracy of perceptions experienced as being from a location outside the body. Although this study therefore did not answer that question, it did provide some clues to the circumstances under which near-death experiences are produced and perhaps, by implication, the etiological mechanisms involved in their production.

We suggest three plausible explanations for this absence of neardeath experiences during induced cardiac arrest: (1) the patients' expectations that their lives would not be in danger, (2) the brief duration of the cardiac arrest, and (3) the amnestic effect of midazolam used to premedicate these patients.

First, participants' reassurance that they would not be in danger of dying may have reduced the likelihood of having a near-death experience. In those near-death experiences that occur in the absence of cardiac arrest, a frequent precipitant appears to be fear of imminent death; indeed, it has been suggested that we call such events feardeath experiences (Stevenson, Cook, and McClean-Rice, 1989-90). To the extent that fear of imminent death may also play a role in neardeath experiences that are associated with cardiac arrest, the safety of the ICD implantation protocol and the reassuring presence of the cardiac electrophysiology team may have inhibited the occurrence of near-death experiences in this population.

Second, the cardiac arrests induced in ICD testing may have been too brief in duration to permit near-death experiences to unfold. There certainly have been reports of near-death experiences in the absence of cardiac arrest (Owens, Cook, and Stevenson, 1990; Stevenson, Cook, 
and McClean-Rice, 1989-1990), and duration of cardiac arrest has been reported to be unrelated to the occurrence of NDEs (van Lommel, van Wees, Meyers, and Elfferich, 2001). However, it is conceivable that patients undergoing ICD testing are not in cardiac arrest long enough to induce NDEs reliably. In EEG studies of patients similar to ours undergoing ICD implantation and testing, the mean time from induction of ventricular fibrillation or last systolic blood pressure to onset of EEG change suggestive of cerebral ischemia was 1011 seconds (Clute and Levy, 1990; deVries, Bakker, Visser, Diephuis, and van Huffelen, 1998), which exceeded the mean duration of cardiac arrest among participants in this study. It is plausible that the ICDs' efficiency terminated cardiac arrest in these patients before neardeath experiences had the time to develop.

Third, the use of the pre-anesthetic sedative midazolam may have made it difficult for participants who did have near-death experiences to remember them following the procedure. The use of general anesthetics in these patients would not in itself preclude subsequent reports of near-death experiences, as many patients describe such experiences under general anesthesia (Kelly, Greyson, and Kelly, 2006). Some patients can recall events that occurred while they were ostensibly anesthetized (Cheek, 1964, 1966; Ghoneim and Block, 1997; Levinson, 1965), and the dissociative anesthetic ketamine may induce events similar to near-death experiences (Jansen, 1997). However, the sedative midazolam that was used to premedicate patients in this study characteristically causes anterograde amnesia independent of its sedative or anesthetic effect (Veselis, Reinsel, and Feshchenko, 1997, 2001). If midazolam does indeed inhibit recall of near-death experiences, in contrast to other sedating medications, that distinction may suggest a biochemical pathway associated with such experiences.

Despite our expectation that medically induced cardiac arrest would provide a controlled setting in which to explore various aspects of near-death experiences, the patients' expectations that they would not be exposed to mortal danger, the brief duration of the cardiac arrest, and/or the amnestic effect of midazolam resulted in the complete absence of near-death experiences in this study. We still believe that medically controlled near-death settings would provide information about these experiences that cannot be obtained by retrospective study of spontaneous occurring experiences. However, future research should utilize procedures in which there is the perception, if not the 
reality, of greater risk to the patient's life, in which the duration of cardiac arrest is prolonged, and in which midazolam is not used routinely. One anecdotal report of a profound near-death experience during a hypothermic circulatory arrest for the excision of a giant basilar artery aneurysm (Sabom, 1998) suggests that that procedure may provide a more promising opportunity for future research into near-death experiences.

\section{References}

Campeau, L. (1976). Grading of angina pectoris. Circulation, 54, 522-523.

Cheek, D. B. (1964). Surgical memory and reaction to careless conversation. American Journal of Clinical Hypnosis, 6, 237-239.

Cheek, D. B. (1966). The meaning of continued hearing sense under general chemoanesthesia: A progress report and report of a case. American Journal of Clinical Hypnosis, 8, 275-280.

Clute, H. L., and Levy, W. J. (1990). Electroencephalographic changes during brief cardiac arrest in humans. Anesthesiology, 73, 821-825.

Cook, E. W., Greyson, B., and Stevenson, I. (1998). Do any near-death experiences provide evidence for the survival of human personality after death? Relevant features and illustrative case reports. Journal of Scientific Exploration, 12, 377-406.

de Vries, J. W., Bakker, P. F. A., Visser, G. H., Diephuis, J. C., and van Huffelen, A. C. (1998). Changes in cerebral oxygen uptake and cerebral electrical activity during defibrillation threshold testing. Anesthesia and Analgesia, 87, 16-20.

Ghoneim, M. M., and Block, R. I. (1997). Learning and memory during general anesthesia: An update. Anesthesiology, 87, 387-410.

Greyson, B. (1983). The Near-Death Experience Scale: construction, reliability, and validity. Journal of Nervous and Mental Disease, 171, 369-375.

Greyson, B. (1998). The incidence of near-death experiences. Medicine and Psychiatry, 1, 92-99.

Greyson, B. (2003). Incidence and correlates of near-death experiences on a cardiac care unit. General Hospital Psychiatry, 25, 269-276.

Holden, J. M. (1988). Visual perception during naturalistic near-death out-of-body experiences. Journal of Near-Death Studies, 7, 107-120.

Holden, J. M. (1989). Unexpected findings in a study of visual perception during the naturalistic near-death-out-of-body experience. Journal of Near-Death Studies, 7, 155-164.

Jansen, K. L. R. (1997). The ketamine model of the near-death experience: A central role for the N-methyl-D-aspartate receptor. Journal of Near-Death Studies, 16, 5-26.

Kelly, E. W., Greyson, B., and Kelly, E. F. (2006). Unusual experiences near death and related phenomena. In E. F. Kelly, E. W. Kelly, A. Crabtree, A. Gauld, M. Grosso, and B. Greyson, Irreducible mind: Toward a psychology for the 21 ${ }^{\text {st }}$ century (pp. 367-421). Lanham, MD: Rowman and Littlefield.

Levinson, B. W. (1965). States of awareness during general anaesthesia: Preliminary communication. British Journal of Anaesthesia, 37, 544-546.

Milne, C. T. (1995). Cardiac electrophysiology studies and the near-death experience. Canadian Association of Critical Care Nurses, 6, 16-19.

Owens, J. E., Cook, E. W., and Stevenson, I. (1990). Features of "near-death experience" in relation to whether or not patients were near death. Lancet, 336, 1175-1177. 
Parnia, S., Waller, D. G., Yeates, R., and Fenwick, P. (2001). A qualitative and quantitative study of the incidence, features and aetiology of near-death experiences in cardiac arrest survivors. Resuscitation, 48, 1149-1156.

Ring, K., and Lawrence, M. (1993). Further evidence for veridical perception during near-death experiences. Journal of Near-Death Studies, 11, 223-229.

Sabom, M. B. (1982). Recollections of death: a medical investigation. New York, NY: Harper and Row.

Sabom, M. B. (1998). Light and death: One doctor's fascinating account of near-death experiences. Grand Rapids, MI: Zondervan.

Schoenbeck, S. B., and Hocutt, G. D. (1991). Near-death experience in patients undergoing cardiopulmonary resuscitation. Journal of Near-Death Studies, 9, 211-218.

Schwaninger, J., Eisenberg, P. R., Schechtman, K. B., and Weiss, A. N. (2002). A prospective analysis of near-death experiences in cardiac arrest patients. Journal of Near-Death Studies, 20, 215-232.

Stevenson, I., Cook, E. W., and McClean-Rice, N. (1989-1990). Are persons reporting "near-death experiences" really near death? A study of medical records. Omega, 20, 45-54.

van Lommel, P., van Wees, R., Meyers, V., and Elfferich, I. (2001). Near-death experience in survivors of cardiac arrest: a prospective study in the Netherlands. Lancet, 358, 2039-2045.

Veselis, R. A., Reinsel, R. A., and Feshchenko, V. A. (1997). The comparative amnestic effects of midazolam, propofol, thiopental, and fentanyl at equisedative concentrations. Anesthesiology, 87, 749-764.

Veselis, R. A., Reinsel, R. A., and Feshchenko, V. A. (2001). Drug-induced amnesia is a separate phenomenon from sedation. Anesthesiology, 95, 896-907.

Währborg, P., and Emanuelsson, H. (1996). The cardiac health profile: Content, reliability and validity of a new disease-specific quality of life questionnaire. Coronary Artery Disease, 7, 823-829. 